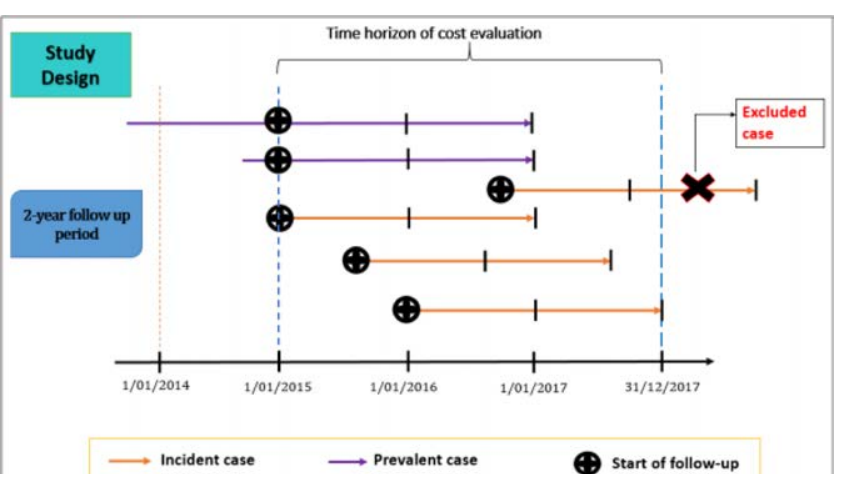

Methods: The present study was carried out with an administrative database that includes all the enrollees in the contributive health scheme for a period of 4 years. It was established an operative definition to identify individuals with the disease in order to make the descriptive analysis and calculation of prevalence. every patient was aged 18 or older on index date. Additionally, as the length of follow-up period was fixed to two years, all patients whose index 14 date had been greater than 1st January 2016 were excluded from the study sample (Figure 1). The variables considered in this part of the study were demografiphc), clinical (Charlson Comorbidity Index) and cost-related variables, which was the outcome variable of the study, this cost was made up of the sum of all medical costs, regardless of whether they were related or not to SLE. Costs were adjusted for inflation, to values in 2017. To evaluate the effect of having SLE vs. not having, on the direct cost in health, propensity scores analysis was used to reduce differences in the baseline characteristics. Three groups were formed based on disease severity: high (patients who had renal failure), medium (patients in intensive care unit at least once but without renal failure) and low (remaining SLE patients) (See table 1).

Table 1. Incremental cost by degree of severity

\begin{tabular}{|c|c|c|c|}
\hline $\begin{array}{l}\text { Degree of } \\
\text { Severity }\end{array}$ & $\begin{array}{l}\text { Average adjust incremen- } \\
\text { tal cost per year (in COP) }\end{array}$ & $\begin{array}{l}\text { Confidence interval con- } \\
\text { struction method }\end{array}$ & $\begin{array}{c}\text { Confidence } \\
\text { interval (95\%) } \\
\text { (in COP) }\end{array}$ \\
\hline \multirow[t]{2}{*}{ High } & \$19,930,931.67 & t-interval & $\begin{array}{l}\$ 16,525,728.01 \\
\$ 23,336,135.32\end{array}$ \\
\hline & & Bootstrap & $\begin{array}{l}\$ 17,088,627.49 \\
\$ 23,068,518.89\end{array}$ \\
\hline \multirow[t]{2}{*}{ Medium } & $\$ 7,248,201.04$ & t-interval & $\begin{array}{l}\$ 2,123,742.99 \\
\$ 12,372,659.09\end{array}$ \\
\hline & & Bootstrap & $\begin{array}{l}\$ 3,460,932.89 \\
\$ 11,688,205.25\end{array}$ \\
\hline \multirow[t]{2}{*}{ Low } & $\$ 885,300.40$ & & $\begin{array}{c}\$ 642,925.6, \$ \\
1,127,675.2\end{array}$ \\
\hline & & Bootstrap & $\begin{array}{c}\$ 688,197.5, \$ \\
1,098,098.2\end{array}$ \\
\hline
\end{tabular}

Results: From 2014 to 2017, 21,993 SLE patients were identified. Women represented $87.4 \%$ of the cases, 5428 patients were selected to make up the sample of SLE patients. the number of patients without diagnosis of SLE was $19,419,540$. From this population was drawn randomly a $10 \%$ size sample, to make up the potential control sample. To estimate the incremental cost of having SLE it was used multivariate regression through a GAM model. The estimated average annual total cost of a patient with SLE was $\$ 6,139,046$ COP vs. non-SLE patient cost of $\$ 4,113,191$ COP. Meanwhile the adjusted incremental cost of SLE vs non-SLE was $\$ 2,025,855$ COP. Subsequently, adjusted incremental cost was estimated taking into account the levels of severity. In the Table 1 are presented the mean values of incremental costs and $95 \%$ confidence intervals.

Conclusion: Although the prevalence of SLE in Colombia is relatively low, the direct costs generated for this disease might be very high. The annual cost for a SLE patient was $\$ 2,025,855$ COP greater than the cost of a non-SLE patient. When considering the severity levels of the disease, it was found a $\$$ $19,930,931.67$ incremental cost estimate for high level of severity. In the medium level, the estimate was $\$ 7,248,201.04$. Meanwhile, a patient in the low severity level had a $\$ 885,300.40$ incremental cost.

References:

[1] Kan HJ, Song X, Johnson BH, Bechtel B, O'Sullivan D, Molta CT. Healthcare utilization and costs of systemic lupus erythematosus in Medicaid. Biomed Res Int. 2013;2013:808391. doi: 10.1155/2013/808391.

\section{SAT0650-HPR WHAT CAN WE LEARN FROM A ROUTINE FRAILTY ASSESSMENT IN RHEUMATOLOGY? A SERVICE EVALUATION OF 170 NEW PATIENT REFERRALS TO A SINGLE RHEUMATOLOGY CENTRE.}

\section{N. Cleaton ${ }^{1}$, J. Bateman ${ }^{1}{ }^{1}$ New Cross Hospital, Heath Town, United Kingdom}

Background: Frailty is common among patients with rheumatological diseases and there is increasing evidence that it is associated with poorer outcomes. Frailty scores worsen during the course of chronic inflammatory arthritis (IA) and connective tissue disease (CTD). Existing tools such as the Rockwood Clinical Frailty Scale (a nine-point scale from 1 very fit- 9 severely frail) allow for rapid frailty assessment by doctors and nurses. Frailty assessment is recommended by NHS England as an important public health opportunity for targeted interventions for both primary and secondary care. We have limited understanding of frailty in rheumatology new patient cohorts, and it is not routinely assessed in most centres. We report the feasibility and utility of frailty scores in patients referred to rheumatology, and association with final diagnosis.

Objectives: This service evaluation assesses the utility and feasibility of implementing the Rockwood Clinical Frailty Score for new patient referrals at a single UK centre.

Methods: New patient assessments at a rheumatology consultant general new patient clinic were prospectively coded over 9-months (March- December 2019). Anonymised coded demographic data included: age, gender, referral source, history of depression, RFCS and clinical diagnosis (coded against established categories, those requiring further tests coded 'awaiting investigations'). RCFS coding was assisted by an online validated pictorial aide memoir for coding.

Results: Of the 181 referrals, $11(6 \%)$ were excluded for incomplete data. The mean age of the remaining 170 patients was 53 years (SD 16.8; range 17-87) predominantly female (123/170; 72\%). Most referrals, $57 \%(97 / 170)$ were from primary care, $23 \%(39 / 170)$ from musculoskeletal integrated triage services, $5 \%$ (9/170) from orthopaedics, 4\% (7/170) from gastroenterology; 18 were from 12 secondary-care specialties. The RCFS was: mean 2.6 (median 2, range 1-7) with depression in $61 / 170(36 \%)$, but no effect of this on mean RCFS (2.6 in both). RCFS increased with age $(70-79, \mathrm{n}=21$, mean $2.9 ;>80, \mathrm{~N}=12,4.3)$. The majority of patients had non-inflammatory (NI) diagnoses (119/170, $70 \%)$ e.g. osteoarthritis, fibromyalgia. Overall 19\% (32) 'inflammatory arthritis' (IA; rheumatoid arthritis, psoriatic, undifferentiated inflammatory arthritis, gout); 4\% (6) were diagnosed with a form of connective tissue disease (undifferentiated CTD; SLE; Sjogren's syndrome); $8 \%$ (13) were awaiting further investigations. Patients in both the $\mathrm{NI}$ and IA category were found to have an average RCFS of 2.6 (SD, NI 1.1; IA 0.9). Patients who received a diagnosis of CTD had an average RCFS of 3.5 (SD 1.8). We identified 30 patients who scored four or more (vulnerable/ at risk) for targeted intervention by therapy and allied health professionals. The RCFS was positively evaluated by clinicians.

Conclusion: RFCS was simple to introduce to our centre and has provided us with additional data to plan our service provision for primary and secondary care support for our cohort. Our new patients with CTD, and who were elderly had higher frailty scores, we found no association between frailty and depression or presence of IA or NI. The RCFS was easy to use and can be integrated into routine clinical practice for new and follow up patients. Further studies are required to support these findings.

References:

[1] Haider, S., Grabovac, I., Berner, C., et al. Frailty in seropositive rheumatoid arthritis patients of working age: a cross-sectional study. Clinical and experimental rheumatology. 2018.

[2] Katz PP, Andrews J, Yazdany J, et al. Is frailty a relevant concept in SLE? Lupus Science \& Medicine 2017;4:e000186.

[3] ALPAY M, CASSEM EH. Diagnosis and treatment of mood disorders in patients with rheumatic disease. Annals of the Rheumatic Diseases 2000;59:2-4

Disclosure of Interests: None declared

DOI: 10.1136/annrheumdis-2020-eular.4665

\section{SAT0651-HPR BARRIERS IN DIAGNOSING RHEUMATOID ARTHRITIS - A FOCUS GROUP STUDY ON THE GENERAL PRACTITIONERS' PERSPECTIVES}

A. S. Lundberg ${ }^{1,2}$, B. A. Esbensen ${ }^{3}$, E. M. Hauge ${ }^{4,5}$, A. De Thurah ${ }^{4,5} .{ }^{1}$ Aarhus University Hospital, Department of Clinical Pharmacology, Aarhus N, Denmark; ${ }^{2}$ Institute of Public Health, Research Unit of General Practice, Aarhus C, Denmark; ${ }^{3}$ Rigshospitalet, Copenhagen Center for Arthritis Research, Center for Rheumatology and Spine Diseases, Copenhagen, Denmark; ${ }^{4}$ Aarhus University Hospital, Department of Rheumatology, Aarhus N, Denmark; ${ }^{5}$ Aarhus University, Department of Clinical Medicine, Aarhus N, Denmark

Background: Early treatment, before three months from symptom onset of rheumatoid arthritis (RA), is essential to increase the likelihood of remission and to 